\title{
An Existence Theorem for a Massive Vector Meson in an External Electromagnetic Field
}

\author{
G. Velo \\ Istituto di Fisica dell'Università, Bologna, \\ Istituto Nazionale di Fisica Nucleare, Sezione di Bologna, Bologna, Italy
}

Received December 22, 1974

\begin{abstract}
The system of Lagrangian equations describing a spin one particle moving in an external electromagnetic field with minimal, dipole and quadrupole interactions is shown to be equivalent to a symmetric hyperbolic system of partial differential equations, to which a standard existence theorem can be applied. The key hypothesis of the treatment is that the derivatives of the electromagnetic field must be sufficiently small. The results cover also the case of noncausal propagation of signals.
\end{abstract}

\section{Introduction}

In a preceding paper [1] (from now on referred to as I) an existence theorem for the system of equations describing a massive vector meson interacting with an external tensor field was proved. The result was obtained by essentially reducing the system of Lagrangian equations to a symmetric hyperbolic system of partial differential equations (PDE) to which standard existence theorem could be applied.

Here we propose to establish an analogous existence theorem for a massive spin one particle in a suitable external electromagnetic field with minimal, dipole and quadrupole couplings. The result is known for minimal interactions [2] and for minimal and dipole interactions [3]. The novelty resides in the treatment of the quadrupole coupling, which is a much less regular case because there can be solutions propagating at a speed greater than the speed of light, that is outside the light cone [4].

The Lagrangian we will start from is essentially that of Bludman and Young [5], but to establish a connection with the Lagrangian used in Ref. [4], we have to add an extra interaction term containing a symmetric external tensor field. In this sense this paper is not only a continuation of I, but it embodies most of the results of $\mathrm{I}$.

The ideas developed here are the same as in I to which we refer both for a description of the situation concerning in general existence theorems and for details about the type of treatment here employed.

In Section 2. we will establish an equivalence theorem between the initial Lagrangian equations and the new equations we will consider. Section 3. contains the proof that, for suitably small external fields, the new system is equivalent to a symmetric hyperbolic system, leading in this way to the existence theorem. A computation of a characteristic determinant is performed in the Appendix. 


\section{The Equivalent System}

Let us consider a complex vector field $V^{\mu}(x)$ in four dimensional space-time coupled to an external electromagnetic field through minimal, dipole and quadrupole interactions. To describe the above physical system we take the following Lagrangian density:

$$
\begin{aligned}
L= & -\frac{1}{2}\left(D_{\mu} V_{\nu}-D_{v} V_{\mu}\right)^{+}\left(D^{\mu} V^{\nu}-D^{\nu} V^{\mu}\right)+m^{2} V_{\mu}^{+} V^{\mu}+i \mu V_{\varrho}^{+} F^{\varrho \sigma} V_{\sigma} \\
& +\frac{q}{2}\left(D_{\mu} V_{v}-D_{v} V_{\mu}\right)^{+} Q_{\lambda}{ }^{\mu \nu} V^{\lambda}+\frac{q^{*}}{2}\left(D_{\mu} V_{\nu}-D_{v} V_{\mu}\right) Q_{\lambda}{ }^{\mu \nu} V^{\lambda^{+}} \\
& -|q|^{2} Q_{\lambda}{ }^{\mu \nu} V^{\lambda^{+}} Q_{\sigma \mu \nu} V^{\sigma}+m^{2} V_{\mu}^{+} T^{\mu \varrho} V_{\varrho}
\end{aligned}
$$

where:

$$
D_{\mu}=\partial_{\mu}+i e A_{\mu}, \quad F_{\mu \nu}=\partial_{\mu} A_{v}-\partial_{v} A_{\mu}, \quad Q_{\lambda \mu \nu}=\partial_{\lambda} F_{\mu \nu},
$$

$A^{\mu}$ is an external electromagnetic potential and $T^{\mu v}$ is an external symmetric tensor field. Here $e$ and $\mu$ are real constants and $q$ is a complex constant measuring respectively the strength of the electric charge, of the dipole moment and of the quadrupole moment. The external fields are supposed to be $C^{\infty}$ functions of the space-time variables. The last term in the expression (2.1) represents a tensor interaction. If this term is zero, Eq. (2.1) reproduces, in the second order formalism the Lagrangian used in Ref. [5], while if:

$$
T_{\mu \nu}=\frac{|q|^{2}}{m^{2}} Q_{\mu \varrho \sigma} Q_{\nu}{ }^{\varrho \sigma} \text {. }
$$

Equation (2.1) reproduces the Lagrangian used in Ref. [4].

Variation with respect to $V^{+}$yields the following Lagrangian equations ${ }^{1}$ :

$$
E^{v}=0
$$

where

$$
\begin{aligned}
E^{\varrho} \equiv & D_{\mu}\left[D^{\mu} V^{\varrho}-D^{\varrho} V^{\mu}-q Q^{\lambda \mu \varrho} V_{\lambda}\right]+m^{2}\left(V^{\varrho}+T^{\varrho \sigma} V_{\sigma}\right) \\
& +i \mu F^{\varrho \sigma} V_{\sigma}+\frac{q^{*}}{2} Q^{\varrho \mu \nu}\left[D_{\mu} V_{\nu}-D_{\nu} V_{\mu}-q Q_{\lambda \mu \nu} V^{\lambda}\right]
\end{aligned}
$$

The characteristic determinant [6] of the second order system of PDE (2.4) is identically zero because of the presence of constraints. To analyze this system we will show that it is equivalent to a new non degenerate system of PDE supplemented by suitable initial conditions.

${ }^{1}$ Our conventions are $g^{\mu v}=\operatorname{diag}(1,-1,-1,-1), \varepsilon^{0123}=1, \varepsilon^{123}=1$, latin indices run from 1 to 3 , greek indices run from 0 to 3 . 
Let us define the expressions:

$$
\begin{aligned}
& N^{i} \equiv D_{\mu} G^{\mu i}+m^{2}\left(V^{i}+T^{i \varrho} V_{\varrho}\right)+i \mu F^{i \varrho} V_{\varrho}+\frac{q^{*}}{2} Q^{i \mu \nu} G_{\mu \nu} \\
& F^{i} \equiv D_{\varrho} H^{\mu i}-\frac{i e}{2} \varepsilon^{e i \sigma \tau} F_{\varrho \sigma} V_{\tau}+q D_{\mu}\left(P_{\lambda}^{\mu i} V^{\lambda}\right) \\
& L \equiv m^{2}\left(D_{\mu} V^{\mu}+D_{\mu} T^{\mu \varrho} V_{\varrho}\right)+\frac{i}{2}(\mu-e) F_{\mu \varrho} G^{\mu \varrho}+\frac{i \mu}{2} q F_{\mu \varrho} Q^{\lambda \mu \varrho} V_{\lambda} \\
& \\
& \quad+\frac{q^{*}}{2} D_{\varrho}\left(Q^{\varrho \mu \nu} G_{\mu \nu}\right)+i \mu\left(\partial_{\nu} F^{v \varrho}\right) V_{\varrho} \\
& S^{i} \equiv m^{2}\left(D^{0} V^{i}-D^{i} V^{0}-q Q^{\lambda 0 i} V_{\lambda}-G^{0 i}\right)
\end{aligned}
$$

where $G^{\mu v}$ is an antisymmetric complex tensor field,

$$
H^{\varrho \lambda} \equiv \frac{1}{2} \varepsilon^{\varrho \lambda \mu v} G_{\mu v}
$$

and

$$
P^{\mu \varrho \lambda} \equiv \frac{1}{2} \varepsilon^{\varrho \lambda \sigma \tau} Q_{\sigma \tau}^{\mu} .
$$

If we take:

$$
G^{\mu \nu}=D^{\mu} V^{v}-D^{v} V^{\mu}-q Q^{\lambda \mu v} V_{\lambda}
$$

and if $V^{\mu}$ is a solution of the system of PDE (2.4), it is easy to check that the $V^{\mu}$ and the $G^{\mu \nu}$ are solutions of the following equations:

$$
\begin{aligned}
& N^{i}=0 \\
& F^{i}=0 \\
& L=0 \\
& S^{i}=0 .
\end{aligned}
$$

There is a converse to this observation, expressed by the following:

Theorem 1. If the $V^{\mu}$ and the $G^{\mu v}$ are solutions of the system (2.10) and if at a specific time the following expressions

$$
S^{i j} \equiv G^{i j}-\left(D^{i} V^{j}-D^{j} V^{i}\right)+q Q^{\lambda i j} V_{\lambda}
$$

and

$$
D_{j} G^{j 0}+m^{2}\left(V^{0}+T^{0} \varrho V_{\varrho}\right)+i \mu F^{0 \varrho} V_{\varrho}+\frac{q^{*}}{2} Q^{0 \varrho \sigma} G_{\varrho \sigma}
$$

are set equal to zero, then the $V^{\mu}$ are solutions of the system (2.4) and the quantities (2.11) and (2.12) will remain zero at all times. 
Proof. The proof is based on two identities which contain the time derivatives of the constraints (2.11) and (2.12) [the expressions (2.11) and (2.12) are called constraints because they do not have the time derivatives of the unknown functions $V^{\mu}$ and $\left.G^{\mu \nu}\right]$. The first is:

$$
D_{0} S_{i j} \equiv-\varepsilon_{i j k} F^{k}+m^{-2}\left(D_{j} S_{i}-D_{i} S_{j}\right)
$$

which is quite easy to check by looking at the explicit expressions for $S_{i j}, F_{k}$, and $S_{i}$. On the other hand, because of the definitions (2.6) and (2.11), the following intermediate identity holds:

$$
\begin{gathered}
D_{v}\left[D_{\mu} G^{\mu \nu}+m^{2}\left(V^{v}+T^{v \varrho} V_{\varrho}\right)+i \mu F^{v \varrho} V_{\varrho}+\frac{q^{*}}{2} Q^{v \varrho \sigma} G_{\varrho \sigma}\right] \\
\equiv L-\frac{i \mu}{2} S_{i j} F^{i j}-i \mu m^{-2} S_{i} F^{i 0} .
\end{gathered}
$$

As a consequence we can deduce the second identity:

$$
\begin{gathered}
D_{0}\left[D_{\mu} G^{\mu 0}+m^{2}\left(V^{0}+T^{0 \varrho} V_{\varrho}\right)+i \mu F^{0 \varrho} V_{\varrho}+\frac{q^{*}}{2} Q^{0 \varrho \sigma} G_{\varrho \sigma}\right] \\
\equiv L-D_{j} N^{j}-i \mu m^{-2} S_{i} F^{i 0}-\frac{i \mu}{2} S_{i j} F^{i j} .
\end{gathered}
$$

Since by hypothesis the $V^{\mu}$ and $G^{\mu v}$ satisfy the system of PDE (2.10), the identities (2.13) and (2.15) become the following differential equations for the constraints (2.11) and (2.12):

and

$$
D_{0} S_{i j}=0
$$

$D_{0}\left[D_{j} G^{j 0}+m^{2}\left(V^{0}+T^{0} \varrho V_{\varrho}\right)+i \mu F^{0} \varrho V_{\varrho}+\frac{q^{*}}{2} Q^{0 \varrho \sigma} G_{\varrho \sigma}\right]=-i \frac{\mu}{2} S_{i j} F^{i j}$.

It is then clear, from Eqs. (2.16) and (2.17), that, if the expression (2.11) and (2.12) are zero in the whole space at a fixed time, they will remain zero at all times. Moreover, since

$$
E^{i} \equiv N^{i}+m^{-2} D_{0} S^{i}-D_{j} S^{j i}
$$

and

$$
E^{0} \equiv\left[D_{j} G^{j 0}+m^{2}\left(V^{0}+T^{0 \varrho} V_{\varrho}\right)+i \mu F^{0 \varrho} V_{\varrho}+\frac{q^{*}}{2} Q^{0 \varrho \sigma} G_{\varrho \sigma}\right]-D_{j} S^{j 0}
$$

it follows that also the initial Lagrangian equations are satisfied.

QED

From now on we will analyze the system of Eq. (2.10), which represents 10 equations in 10 unknown functions. We rewrite it in a synthetic form as:

$$
\Gamma^{\mu} \partial_{\mu} u+D u=0
$$


where

$$
u=\left(G^{01}, G^{02}, G^{03}, H^{01}, H^{02}, H^{03}, V^{0}, V^{1}, V^{2}, V^{3}\right)
$$

and the equations in the expression (2.20) are written in the same order as the Eq. (2.10). The explicit form of the $10 \times 10$ matrices $\Gamma^{\mu}$ and $D$ can be read directly from Eq. (2.10). The matrices $\Gamma^{\mu}$ are not hermitian and therefore the system $(2.20)$ is not symmetric hyperbolic.

The usual definition of symmetric hyperbolicity refers to real systems of PDE (Ref. [6], p. 593, Ref. [7], p. 16). We will employ the same terminology and the same existence theorems also for the complex case, where the obvious substitute for the notion of symmetricity is the notion of hermiticity. The justification for this extensive use is that we can go from a complex to a real system of PDE by splitting equations and unknown functions into real and imaginary parts. Then an $N$ dimensional hermitian matrix coefficient of the complex system becomes a $2 N$ dimensional symmetric matrix coefficient of the corresponding real system, and in this passage the positivity property of a matrix is preserved.

Coming back to the system (2.20), what we have gained by the reduction expressed by Theorem 1 is the elimination of the degeneracy of the initial system (2.4). In fact, as will be shown in the next Section and in the Appendix, the characteristic determinant of the new system (2.20) is not identically zero.

\section{The Existence Theorem}

It is possible to symmetrize the system $(2.10)$ by taking suitable linear combinations of the initial equations, that is of the expressions (2.6). We have the following:

Theorem 2. Provided the matrix $C=\left(c_{i k}\right)$ with

$$
c_{i k}=m^{2}\left(\delta_{i k}-T_{i k}\right)+|q|^{2} Q_{i 0 j} Q_{k}{ }^{j j}
$$

is invertible, the system (2.10) is equivalent to the new system:

$$
\begin{aligned}
& N^{i}+q m^{-2} Q_{j}{ }^{0 i} S^{j}=0 \\
& F^{i}-q m^{-2} P_{j}{ }^{0 i} S^{j}=0 \\
& L-q^{*} Q_{00 i} N^{i}-T_{0 i} S^{i}=0 \\
& -b_{k}^{i} S^{k}+q^{*} Q_{0 j}^{i} N^{j}=0
\end{aligned}
$$

where:

$$
b_{i k}=\delta_{i k}-T_{i k}
$$

Moreover if we rewrite the new system (3.2) in the form:

$$
A^{\mu} \partial_{\mu} u+R u=0
$$


with $u$ given by the expression (2.21), then the $10 \times 10$ matrices $A^{\mu}$ can be explicitly computed and turn out to be hermitian.

Proof. Since one passes from the system (2.10) to the system (3.2) by a linear transformation, it is obvious that there is a $10 \times 10$ matrix $M$ such that

$$
A^{\mu}=M \Gamma^{\mu} \quad R=M D
$$

Its explicit form can be easily seen from the structure of the system (3.2):

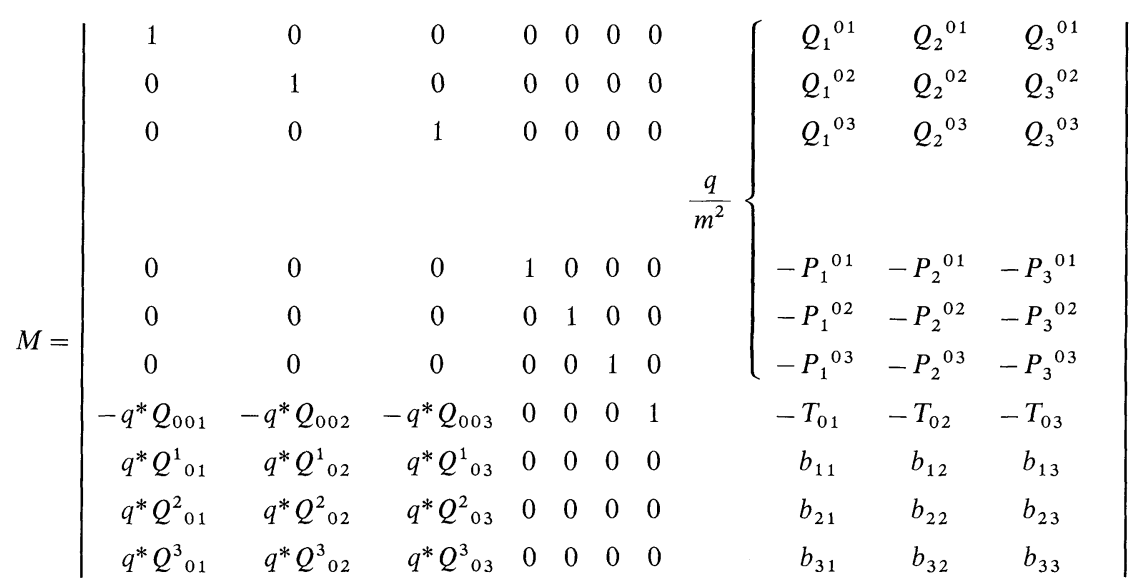

The determinant of $M$ can now be computed in the following way. If we denote by $m_{j k}$ the matrix elements of $M$, the new matrix obtained from $M$ by leaving unchanged the first 7 rows and by substituting for $m_{7+k, j}(k=1,2,3 ; j=1,2, \ldots, 10)$ $m_{7+k, j}+q^{*} \Sigma_{1 i}^{3} Q_{k 0 i} m_{i, j}$, has the same determinant of $M$. The last three rows of the new matrix are:

$$
\left|\begin{array}{llllllll}
0 & 0 & 0 & 0 & 0 & 0 & 0 & \\
0 & 0 & 0 & 0 & 0 & 0 & 0 & m^{-2} C \\
0 & 0 & 0 & 0 & 0 & 0 & 0 &
\end{array}\right|
$$

By developing now the new matrix along the 7 th column we see that:

$$
\operatorname{det} M=m^{-6} \operatorname{det} C \text {. }
$$

The characteristic matrix of the system (3.4) $A^{\mu} n_{\mu}$ can be, with some patience, calculated ( $n_{\mu}$ is a four-vector). If we denote by $a_{i j}=a_{i j}(n)$ its matrix elements, it results that

$$
a_{i j}\left(n^{*}\right)^{*}=a_{j i}(n)
$$


and therefore that the $A^{\mu}$ are hermitian matrices. The explicit expression for the $a_{i j}$ is the following:

$$
\begin{array}{rlrl}
a_{i j}= & n_{0} \delta_{i j} & i=1,2,3 ; \\
n_{0} \delta_{i j} & i=4,5,6 ; \\
\varepsilon_{i, j-3, k} n^{k} & i=1,2,3 ; \\
q n_{0} Q_{j-7,{ }^{0 i}} & i=1,2,3 ; \\
q n_{k} P_{j-7, i-3},^{2} & i=4,5,6 ; \\
m^{2} n_{0} b_{i-7, j-7} & i=8,9,10 ; \\
a_{7 i}=-q^{*} n_{j} Q^{j 0 i} & i=1,2,3 \\
q^{*}\left(n_{\mu} P^{\mu 0, i-3}+n_{j} P_{0}^{j, i-3}\right) & i=4,5,6 \\
& m^{2}\left[n_{0}\left(1+T_{00}\right)+2 n_{i} T^{0 i}\right] & i=7 \\
& m^{2} n_{j} b_{j, i-7} & i=8,9,10 .
\end{array}
$$

The matrix elements not explicitly contained in (3.9) can be recovered via the equality (3.8).

Q.E.D.

With the explicit expression for the matrix $A^{\mu} n_{\mu}$ we can compute its determinant which is called characteristic determinant. The calculation is performed in the Appendix and yields the following result:

$$
\begin{aligned}
\operatorname{det}\left(A^{\mu} n_{\mu}\right)= & n_{0}^{4} n^{2}(\operatorname{det} C)\left\{m^{2}\left(n^{2}\right)^{2}+|q|^{2}\left(n_{\mu} n_{\varrho} Q^{\mu \varrho \sigma}\right)\left(n_{\tau} n_{\lambda} Q^{\tau \lambda}{ }_{\sigma}\right)\right. \\
& \left.+n^{2}\left[m^{2} n \cdot T \cdot n-\frac{|q|^{2}}{2}\left(n_{\varrho} Q^{\varrho}{ }_{\sigma \tau}\right)\left(n_{\mu} Q^{\mu \sigma \tau}\right)\right]\right\} .
\end{aligned}
$$

Concerning the existence of solutions we are now in a position to prove the following:

Theorem 3. Let the matrix $C$ be positive and bounded away from zero uniformly in space-time, let the quantity

$$
m^{2}\left(1+T_{00}\right)-|q|^{2} \sum_{i<j}\left(Q^{0 i j}\right)^{2}
$$

be positive and bounded away from zero uniformly in space-time, let the external fields be infinitely differentiable functions of the space-time variables. Then the system (3.4) is a symmetric hyperbolic system and it has for infinitely differentiable initial data a unique infinitely differentiable solution.

Proof. The existence result follows by a standard theorem once it is proved that under the stated conditions the system (3.4) is symmetric hyperbolic (Ref. [6], p. 669, Ref. [7], p. 89). To establish the symmetric hyperbolicity the only point which remains to be checked is the positivity of $A^{0}$. From the explicit expression for $A^{\mu} n_{\mu}$ in (3.9) one finds that:

$$
\begin{aligned}
\left(u, A^{0} u\right)= & \left|G^{0 i}+q Q_{j}{ }^{0 i} V^{j}\right|^{2}+\left|H^{0 i}+q P_{0}{ }^{0 i} V^{0}\right|^{2} \\
& +\left[m^{2}\left(1+T_{00}\right)-|q|^{2}\left(P_{0}{ }^{0 i}\right)^{2}\right] V^{0+} V^{0}+c_{i j} V_{i}^{+} V_{j}
\end{aligned}
$$


By relation (2.8) it follows that:

$$
\Sigma_{i}\left(P_{0}^{0 i}\right)^{2}=\sum_{i<j}\left(Q^{0 i j}\right)^{2}
$$

Therefore, by the hypothesis of the theorem, $A^{0}$ is uniformly bounded from below in the whole space-time by a positive number.

Q.E.D.

We suppose from now on that the hypothesis of Theorem 3 are satisfied. The space-like surfaces of the hyperbolic system (3.4) are defined by the condition that $A^{\mu} \sigma_{\mu}>0$, where $\sigma^{\mu}$ is the normal to the surface and $\sigma^{0}$ is taken to be positive. We will say that $\left(n^{0}, \boldsymbol{n}\right)$ is, for $\boldsymbol{n}$ fixed, the largest solution to Eq. (3.10) if $\left(n^{0}, \boldsymbol{n}\right)$ is the solution to Eq. (3.10) with the largest $n^{0}$ for a given $n$. Then it is easy to see that $\left(\sigma^{0}, \boldsymbol{\sigma}\right)$ is normal to a space-like surface if and only if $\sigma^{0}>n^{0}$ where $\left(n^{0}, \boldsymbol{n}\right)$ is the largest solution to Eq. (3.10) with $\boldsymbol{n}=\boldsymbol{\sigma}$. Moreover the quotients $\frac{n^{0}}{|\boldsymbol{n}|}$, where $\left(n^{0}, \boldsymbol{n}\right)$ belongs to the family of the largest solutions to Eq. (3.10), represent the maximal speed of propagation of signals in the theory. It is easy to have situations in which such a speed is greater than the speed of light in vacuum [4].

Finally the existence theorem extends through Theorems 1 and 2 to the initial system (2.4), provided we impose that at the initial time the expressions (2.11) and (2.12) are set equal to zero. These restrictions on the initial data can be easily satisfied. In fact, because of the hypothesis of Theorem 3 , the quantities $G^{i j}$ and $V^{0}$ can be immediately expressed as linear functions of the $G^{0 i}$ and $V^{j}$ and of their space derivatives.

\section{Appendix}

Here we want to compute the determinant of the matrix $A \equiv A^{\mu} n_{\mu}$, where the matrix elements $a_{i j}$ are given by Eqs. (3.8) and (3.9).

Let us consider the new matrix $A_{1}$ in which the elements of the first six rows are the same as those of $A$ and in which the remaining ones are obtained in the following way. The elements of the 7 th row are:

$$
a_{7 i}-\sum_{1}^{3} a_{7+j, i} \frac{n_{j}}{n_{0}}
$$

and those of the $(7+k)$ th rows $(k=1,2,3)$ are:

$$
a_{7+k, i}-q^{*} \sum_{1}^{3} Q^{k}{ }_{0 j} a_{j i}
$$

Obviously $A_{1}$ has the same determinant as $A$. Let us perform now on the transpose of the matrix $A_{1}$ the same operations we performed on $A$. We leave unchanged the first six rows of $A_{1}^{T}$ while we change the $7 \mathrm{th}, 8 \mathrm{th}, 9$ th and $10 \mathrm{th}$ according to the rules (A.1) and (A.2), where the $a_{i j}$ are replaced by the matrix elements of $A_{1}^{T}$, while the coefficients of the linear combinations remain the same. The resulting 
matrix, which has the same determinant as $A$, is the following:

$$
\left|\begin{array}{cccccccccc}
n_{0} & 0 & 0 & 0 & n_{3} & -n_{2} & 0 & 0 & 0 & 0 \\
0 & n_{0} & 0 & -n_{3} & 0 & n_{1} & 0 & 0 & 0 & 0 \\
0 & 0 & n_{0} & n_{2} & -n_{1} & 0 & 0 & 0 & 0 & 0 \\
0 & -n_{3} & n_{2} & n_{0} & 0 & 0 & \frac{\alpha^{1}}{n_{0}} & 0 & 0 & 0 \\
n_{3} & 0 & -n_{1} & 0 & n_{0} & 0 & \frac{\alpha^{2}}{n_{0}} & 0 & 0 & 0 \\
-n_{2} & n_{1} & 0 & 0 & 0 & n_{0} & \frac{\alpha^{3}}{n_{0}} & 0 & 0 & 0 \\
0 & 0 & 0 & \frac{\alpha^{1 *}}{n_{0}} & \frac{\alpha^{2 *}}{n_{0}} & \frac{\alpha^{3^{*}}}{n_{0}} & \frac{d}{n_{0}} & 0 & 0 & 0 \\
0 & 0 & 0 & 0 & 0 & 0 & 0 & & & \\
0 & 0 & 0 & 0 & 0 & 0 & 0 & n_{0} C & \\
0 & 0 & 0 & 0 & 0 & 0 & 0 & &
\end{array}\right| .
$$

Here

$$
\begin{aligned}
\alpha^{i} & =q n_{e} n_{\mu} P^{e \mu i}, \\
d & =m^{2}(z+n \cdot T \cdot n), \\
z & =n^{2},
\end{aligned}
$$

and $C$ is the $3 \times 3$ matrix (3.1).

To further simplify the computation of the determinant, we perform on the matrix (A.3) the following operations. We add to the 4 th row the 2 nd multiplied by $\frac{n_{3}}{n_{0}}$ and the 3 rd multiplied by $-\frac{n_{2}}{n_{0}}$, to the 5 th row the 1 st multiplied by $-\frac{n_{3}}{n_{0}}$ and the 3 rd multiplied by $\frac{n_{1}}{n_{0}}$, to the 6 th row the 1 st multiplied by $\frac{n_{2}}{n_{0}}$ and the 2nd multiplied by $-\frac{n_{1}}{n_{0}}$. The only changes in the matrix involve the first 6 rows and columns, that we are going to rewrite:

$$
\left|\begin{array}{cccccc}
n_{0} & 0 & 0 & 0 & n_{3} & -n_{2} \\
0 & n_{0} & 0 & -n_{3} & 0 & n_{1} \\
0 & 0 & n_{0} & n_{2} & -n_{1} & 0 \\
0 & 0 & 0 & \frac{z+n_{1}^{2}}{n_{0}} & \frac{n_{1} n_{2}}{n_{0}} & \frac{n_{1} n_{3}}{n_{0}} \\
0 & 0 & 0 & \frac{n_{1} n_{2}}{n_{0}} & \frac{z+n_{2}^{2}}{n_{0}} & \frac{n_{2} n_{3}}{n_{0}} \\
0 & 0 & 0 & \frac{n_{1} n_{3}}{n_{0}} & \frac{n_{2} n_{3}}{n_{0}} & \frac{z+n_{3}^{2}}{n_{0}}
\end{array}\right| .
$$


Therefore the determinant of the matrix (A.3) becomes:

$$
n_{0}^{2}(\operatorname{det} C) \operatorname{det}\left|\begin{array}{cccc}
z+n_{1}^{2} & n_{1} n_{2} & n_{1} n_{3} & \alpha^{1} \\
n_{1} n_{2} & z+n_{2}^{2} & n_{3} n_{3} & \alpha^{2} \\
n_{1} n_{3} & n_{2} n_{3} & z+n_{3}^{2} & \alpha^{3} \\
\alpha^{1^{*}} & \alpha^{2 *} & \alpha^{3^{*}} & d
\end{array}\right| .
$$

The $4 \times 4$ determinant contained in (A.8) can be directly computed. The result is:

$$
z\left[d z n_{0}^{2}-\boldsymbol{\alpha}^{*} \cdot \boldsymbol{\alpha} n_{0}^{2}+(\boldsymbol{\alpha} \cdot \boldsymbol{n})^{*}(\boldsymbol{\alpha} \cdot \boldsymbol{n})\right] .
$$

By the definitions (A.4)-(A.6), it follows that the determinant of $A^{\mu} n_{\mu}$ is:

$$
n_{0}^{4} n^{2}\left\{n^{2} m^{2}\left(n^{2}+n \cdot T \cdot n\right)+|q|^{2}\left(n_{\varrho} n_{\varrho} P^{\mu \varrho \sigma}\right)\left(n_{\tau} n_{\lambda} P_{\sigma}^{\tau \lambda}\right)\right\}(\operatorname{det} C) \text {. }
$$

The final expression (3.10) is obtained by the expression (A.10) using the definition (2.8).

\section{References}

1. Velo, G.: An existence theorem for a massive spin one particle in an external tensor field (in press on the Annales de l'Institut H. Poincaré, 1975)

2. Wightman, A.S.: In Salam,A., Wigner,E.P. (Eds.): Aspects of quantum theory. Cambridge: University Press 1972

3. Bellissard, J.: Quantized fields in external field. II. Existence theorem (preprint)

4. Velo, G., Zwanziger, D.: Phys. Rev. 188, 2218 (1969)

5. Bludman, S., Young, J.: Phys. Rev. 131, 2326 (1963)

6. For the general ideas and the terminology involved in the theory of Partial Differential equations we refer to the book by Courant, R., Hilbert, D.: Methods of mathematical physics, Vol. 2, especially Chapter 6. New York: Wiley-Interscience Inc. 1962

7. Lax,P.D.: Lectures on hyperbolic partial differential equations, mimeographed notes. Stanford University, Spring-Summer 1963

Communicated by A. S. Wightman

G. Velo

Istituto di Fisica

dell' Università

Via Irnerio 46

I-40126 Bologna, Italy 